\title{
LONG PERIODIC ORBITS OF THE TRIANGLE MAP
}

\author{
MANNY SCAROWSKY AND ABRAHAM BOYARSKY ${ }^{1}$
}

\begin{abstract}
Let $\tau:[0,1] \rightarrow[0,1]$ be defined by $\tau(x)=2 x$ on $[0,1 / 2]$ and $\tau(x)=$ $2(1-x)$ on $[1 / 2,1]$. We consider $\tau$ restricted to the domain $D_{N}=\left\{2 a / p^{N}, N \geqslant\right.$ $\left.1,0 \leqslant 2 a \leqslant p^{N},(a, p)=1\right\}$ where $p$ is any odd prime. Let $k \geqslant 1$ be the minimum integer such that $p^{N} \mid 2^{k} \pm 1$. Then there are $\left((\mathrm{p}-1) \cdot p^{N-1}\right) / 2 k$ periodic orbits of $\left.\tau\right|_{D_{N}}$. having equal length, and there are $k$ points in each orbit. Furthermore, the proportion of points in any of these periodic orbits which lie in an interval $(c, d)$ approaches $d-c$ as $p^{N-1} \rightarrow \infty$. An application to the irreducibility of certain nonnegative matrices is given.
\end{abstract}

1. Introduction. Let $\tau:[0,1] \rightarrow[0,1]$ denote the triangle map, defined by

$$
\tau(x)= \begin{cases}2 x, & 0 \leqslant x \leqslant 1 / 2 \\ 2(1-x), & 1 / 2 \leqslant x \leqslant 1\end{cases}
$$

This map, together with its topological conjugate map $g(x)=4 x(1-x)$, have in recent years played an important role in understanding the dynamical behaviour of one-dimensional models of certain nonlinear phenomena [1, 2]. These maps have periodic orbits of all periods and aperiodic orbits which approach neither an equilibrium nor a periodic orbit. "From a practical viewpoint it is probably impossible to distinguish an aperiodic orbit from one with a very long period" $[2, \mathrm{p}$. 107]. The purpose of this note is to give mathematical substance to this conjecture, by proving the existence of long periodic orbits which "exhibit" Lebesque measure, the unique absolutely continuous measure invariant under $\tau$. We say that a periodic orbit "exhibits" a measure $\mu$ on $[0,1]$ if the proportion of points in the orbit that lie in an interval $(c, d)$ approaches $\mu([c, d])$.

As an application of the long periodic orbits that we shall display, we shall be able to prove the reducibility or irreducibility of certain large, sparse, nonnegative matrices.

2. Main result. Consider $\tau$ with the domain $D=D_{p^{N}}=\left\{0<2 x / p^{N}<1,(x, p)\right.$ $=1\}$, where $p$ is an odd prime, $N$ is an integer $\geqslant 1$, and $(x, p)=1$ denotes the fact that $x$ and $p$ are relatively prime. Let $f=\left.\tau\right|_{D}: D \rightarrow[0,1]$. It is easy to show that $f$ : $D \rightarrow D$, and that $|D|$, the number of points in $D$, is equal to $((p-1) / 2) \cdot p^{N-1}$.

Received by the editors September 21, 1984.

1980 Mathematics Subject Classification. Primary 28D05; Secondary 15 A36.

${ }^{1}$ Research supported by NSERC Grant \# A9072 and an FCAC grant from the Quebec Department of Education. 
TheOREM 1. The map $f: D \rightarrow D$ is an isomorphism and induces a partition of $D$ into disjoint periodic orbits of equal length. Let $k=k\left(p^{N}\right)$ be the minimum integer $\geqslant 1$ such that $p^{N} \mid 2^{k} \pm 1$. Then the number of points in each orbit is $k$, and the number of periodic orbits is $\left((p-1) \cdot p^{N-1}\right) / 2 k$.

Proof. Using the facts that

$$
f\left(2 x / p^{N}\right)=2(\text { even integer }) / p^{N}, \quad \text { if } 2 x / p^{N}<\frac{1}{2},
$$

and

$$
f\left(2 x / p^{N}\right)=2(\text { odd integer }) / p^{N}, \quad \text { if } 2 x / p^{N}>\frac{1}{2},
$$

it is easy to see that $f$ is an isomorphism.

Now, if $2 x / p^{N}$ has period $l$, then $A \pm 2^{l}\left(2 x / p^{N}\right)=2 x / p^{N}, A \in \mathbf{Z}$. This implies that $p^{N} \mid 2^{l} \pm 1$, and it follows that $l$ must be a multiple of $k$. Note that

$$
k=k\left(p^{N}\right)= \begin{cases}\lambda\left(p^{N}\right) / 2 & \text { if } \lambda\left(p^{N}\right) \text { is even, } \\ \lambda\left(p^{N}\right) & \text { if } \lambda\left(p^{N}\right) \text { is odd, }\end{cases}
$$

where $\lambda\left(p^{N}\right)$ is the order of $2\left(\bmod p^{N}\right)$. This follows from the fact that $\lambda\left(p^{N}\right)$ is the minimum integer $\geqslant 1$ such that $p^{N} \mid 2^{\lambda\left(p^{N}\right)}-1$ : since $p^{N} \mid 2^{2 k}-1$, we have that $k \leqslant \lambda\left(p^{N}\right) \leqslant 2 k$, i.e., $1 \leqslant 2 k / \lambda\left(p^{N}\right) \leqslant 2$. Since $2 k / \lambda\left(p^{N}\right)$ is an integer, $\lambda\left(p^{N}\right)=$ $k, 2 k$. Thus $k=\lambda\left(p^{N}\right), \lambda\left(p^{N}\right) / 2$. Clearly, if $\lambda\left(p^{N}\right)$ is odd the first case occurs, and if $\lambda\left(p^{N}\right)$ is even, the second case occurs.

We now show that if $2 x_{1} / p^{N} \in D$, then $f^{k}\left(2 x_{1} / p^{N}\right)=\left(2 x_{1} / p^{N}\right)$. It is easy to verify that

$$
\tau^{m}(x)= \begin{cases}2^{m} x-i, & x \in\left[i / 2^{m},(i+1) / 2^{m}\right], i \text { even } \\ -2^{m} x+i+1, & x \in\left[i / 2^{m},(i+1) / 2^{m}\right], i \text { odd }\end{cases}
$$

This follows from the continuity of $\tau^{m}$, which has slope $\pm 2^{m}$, and from the fact that $\tau^{m}(0)=\tau^{m}(1)=0$. Let $x=2 x_{1} / p^{N}$. Then $i$ is determined by $i / 2^{m}<2 x_{1} / p^{N}<$ $(i+1) / 2^{m}$, i.e., $i p^{N}<2^{m+1} x_{1}<(i+1) p^{N}, 0<2^{m+1} x_{1}-i p^{N}<p^{N}$, or $0<$ $2^{m+1} x_{1} / p^{N}-i<1$. Therefore, $i=\left[2^{m+1} x_{1} / p^{N}\right]$. Using (1), we can determine $\tau^{k}\left(2 x_{1} / p^{N}\right)$

$$
\begin{aligned}
\tau^{k}\left(\frac{2 x_{1}}{p^{N}}\right) & =\frac{2^{k+1} x_{1}}{p^{N}}-\left[\frac{2^{k+1} x_{1}}{p^{N}}\right] \text { if }\left[\frac{2^{k+1} x_{1}}{p^{N}}\right] \text { is even, } \\
\tau^{k}\left(\frac{2 x_{1}}{p^{N}}\right) & =\frac{-2^{k+1} x_{1}}{p^{N}}+\left[\frac{2^{k+1} x_{1}}{p^{N}}\right]+1 \text { if }\left[\frac{2^{k+1} x_{1}}{p^{N}}\right] \text { is odd. }
\end{aligned}
$$

But

$$
2^{k+1} x_{1} / p^{N}=\left(2^{k+1} \pm 2\right) x_{1} / p^{N} \mp 2 x_{1} / p^{N}
$$


If $p^{N} \mid 2^{k}+1$, then $\left[2^{k+1} x_{1} / p^{N}\right]=\left(2^{k+1}+2\right) x_{1} / p^{N}-1$ is odd, and using (3), we obtain $\tau^{k}\left(2 x_{1} / p^{N}\right)=2 x_{1} / p^{N}$. Similarly, if $p^{N} \mid 2^{k}-1,\left[2^{k+1} x_{1} / p^{N}\right]$ is even and from (2) we obtain $\tau^{k}\left(2 x_{1} / p^{N}\right)=2 x_{1} / p^{N}$. Thus, the period of $2 x_{1} / p^{N}$ is exactly $k$ and the number of periodic orbits is $|D| / k=\left((p-1) \cdot p^{N-1}\right) /(2 \cdot k)$. Q.E.D.

REMARK. If for some positive integer $m^{\prime}, p^{m^{\prime}} \| 2^{k(p)} \pm 1$, i.e., the division is exact, then $k(p)=k\left(p^{2}\right)=\cdots=k\left(p^{m^{\prime}}\right)$.

In Table 1, we display for $N=1$, the period $k$ and the number of the periodic orbits for $3 \leqslant p \leqslant 100$. Note that $k=k(p)$ satisfies $1 \leqslant k \leqslant(p-1) / 2$ and $k \mid(p-1) / 2$. Since $p \mid 2^{k} \pm 1,2^{k} \geqslant p-1$, and $k \geqslant \log (p-1)$. Therefore, $k(p) \rightarrow$ $\infty$ as $p \rightarrow \infty$.

\section{TABLE 1}

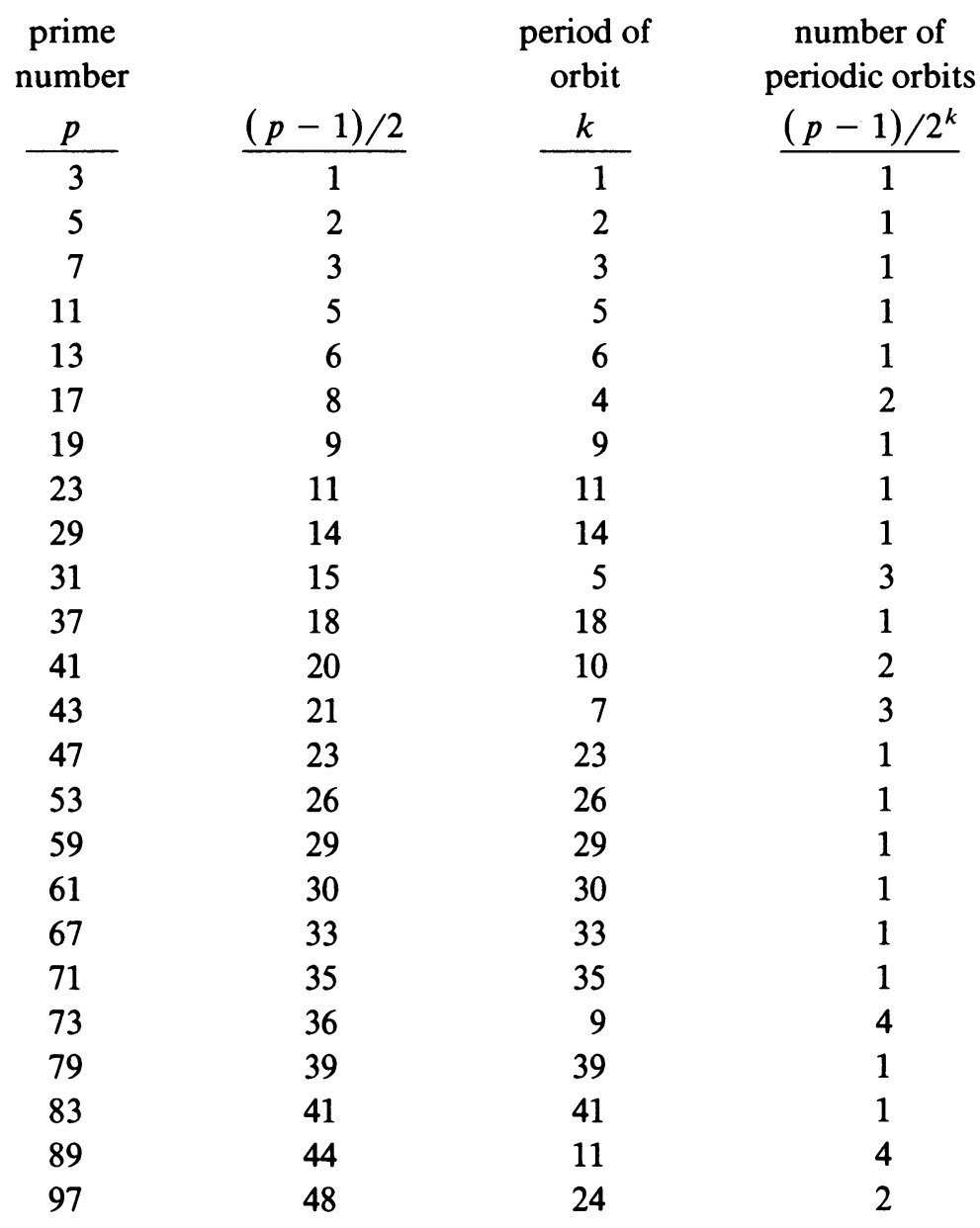

LEMMA 1. Let $p \mid 2^{\lambda(p)}-1$, where $\lambda(p) \geqslant 1$ is minimal and assume $p^{m^{\prime}} \| 2^{\lambda(p)}-1$. Then for any $N \geqslant m^{\prime}, p^{N} \mid 2^{\lambda^{\prime}}-1$ iff $\lambda^{\prime}$ is a multiple of $\lambda(p) p^{N-m^{\prime}}$, and the minimum value of $\lambda^{\prime}>0$ such that $p^{N} \mid 2^{\lambda^{\prime}}-1$ equals $\lambda(p) p^{N-m^{\prime}}$. 
Proof. Clearly the result is true for $N=m^{\prime}$. Assume the result is true for $N=l>m^{\prime}$. Then $p^{l+1} \mid 2^{\lambda^{\prime}}-1$ implies $p^{l} \mid 2^{\lambda^{\prime}}-1$ implies $\lambda^{\prime}=a \cdot \lambda(p) p^{l-m^{\prime}}$, and $p^{l+1} \mid 2^{a \lambda(p) \cdot p^{\prime-m^{\prime}}}-1$. But

$$
2^{a \lambda(p) \cdot p^{l-m^{\prime}}}-1=\left(1+\delta p^{m^{\prime}}\right)^{a p^{\prime-m^{\prime}}}-1=a \delta p^{l}+\text { multiple of } p^{l+1},
$$

where $(\delta, p)=1$. Thus $p \mid a$ and the lemma is proved. Q.E.D.

RemarK. For $p<500,000, m^{\prime}>1$ only for $p=1093$ and $p=3511$, in which case $m^{\prime}=2$.

THEOREM 2. The proportion of points in a fixed period orbit which lies in an interval $(c, d)$ approaches $d-c$ as $p^{N-m^{\prime}} \rightarrow \infty$.

Proof. Let $2^{m+1} x_{1}=q_{m} p^{N}+r_{m}$, where $q_{m}, r_{m} \in \mathbf{Z}, 0<r_{m}<p^{N}$ and $2 x_{1} / p^{N} \in$ $D$. For technical reasons we let $m$ vary from 1 to $2 k$. Then $2^{m+1} x_{1} / p^{N}=q_{m}+r_{m} / p^{N}$ and

$$
\tau^{m}\left(\frac{2 x}{p^{N}}\right)= \begin{cases}r_{m} / p^{N}, & r_{m} \text { even } \\ \left(p^{N}-r_{m}\right) / p^{N}, & r_{m} \text { odd }\end{cases}
$$

Let $\#\{\}$ denote the number of points in the set \{\} . Then

$$
\begin{aligned}
\#\{m: & \left.\tau^{m}\left(2 x_{1} / p^{N}\right) \in(c, d), 1 \leqslant m \leqslant 2 k\right\} \\
= & \#\left\{m: r_{m} / p^{N} \in(c, d), r_{m} \text { even; }\left(p^{N}-r_{m}\right) / p^{N} \in(c, d), r_{m} \text { odd }\right\} \\
= & \#\left\{m: p^{N} c<r_{m}<p^{N} d, r_{m} \text { even; } p^{N} c<p^{N}-r_{m}<p^{N} d, r_{m} \text { odd }\right\} .
\end{aligned}
$$

Now, by Lemma $1, k=k\left(p^{N}\right)=k^{\prime} \cdot p^{N-m^{\prime}}$, where $k^{\prime}=k(p)$ and $N \geqslant m^{\prime}$. Let $r_{1}^{\prime}, \ldots, r_{k^{\prime \prime}}^{\prime}$ be the residue classes of the $r_{i}(\bmod p)$, i.e., the residue classes when $N=1$. Note that $k^{\prime \prime}=k^{\prime}$ or $2 k^{\prime}$. Then it is easy to see that $r_{m}=p^{m^{\prime}} i+r_{j}^{\prime}$, $0 \leqslant i<p^{N-m^{\prime}}, 1 \leqslant j \leqslant 2 k^{\prime}$, for $N \geqslant m^{\prime}$. Hence, the right-hand side of (4) equals

$$
\begin{aligned}
\#\{m: & p^{N} c<p^{m^{\prime}} i+r_{j}^{\prime}<p^{N} d, i+r_{j}^{\prime} \text { even; } \\
& \left.p^{N} c<p^{N}-p^{m^{\prime}} i-r_{j}^{\prime}<p^{N} d, i+r_{j}^{\prime} \text { odd }\right\} \\
= & \sum_{j=1}^{2 k^{\prime}} \#\left\{i: p^{N} c-r_{j}^{\prime}<p^{m^{\prime}} i<p^{N} c-r_{j}^{\prime}, i+r_{j}^{\prime} \text { even }\right\} \\
+ & \sum_{j=1}^{2 k^{\prime}} \#\left\{i: p^{N} c+r_{j}^{\prime}-p^{N}<-p^{m^{\prime}} i<p^{N} d+r_{j}^{\prime}-p^{N}, i+r_{j}^{\prime} \text { odd }\right\} .
\end{aligned}
$$

Since the number of even (odd) integers in an interval $(e, f)$ is $((f-e) / 2)+O(1)$, the right-hand side of (5) sums to

$$
\begin{aligned}
& \sum_{j=1}^{2 k^{\prime}}\left(\frac{p^{N-m^{\prime}}(d-c)}{2}+O(1)\right)+\sum_{j=1}^{2 k^{\prime}}\left(\frac{p^{N-m^{\prime}}(d-c)}{2}+O(1)\right) \\
& =2 k^{\prime} p^{N-m^{\prime}}(d-c)+O\left(k^{\prime}\right) .
\end{aligned}
$$


Dividing through by $2 k=2 k^{\prime} p^{N-m^{\prime}}, N \geqslant m^{\prime}$, we get

$$
\#\left\{m: \tau^{m}\left(2 x_{1} / p^{N}\right) \in(c, d), 1 \leqslant m \leqslant k\right\} / k=(d-c)+O\left(1 / p^{N-m^{\prime}}\right) .
$$

The right-hand side approaches $d-c$ as $p^{N-m^{\prime}} \rightarrow \infty$. Q.E.D.

REMARK. For $p$ fixed, we need $N \rightarrow \infty$. For $p$ increasing it is enough that $N=N(p)>m^{\prime}$, as $p \rightarrow \infty$.

COROLLARY 1. Let $h$ be a bounded, continuous function on $[0,1]$. Then

$$
\lim _{\substack{p^{N-m^{\prime} \rightarrow \infty} \\ x \in D}} \frac{1}{k} \sum_{i=1}^{k} h\left(\tau^{i}(c)\right)=\int_{0}^{1} h(x) d x .
$$

Proof. Given any $\varepsilon>0$, Theorem 2 implies that

$$
\left|\frac{1}{k} \sum_{i=1}^{k} \chi_{(c, d)}\left(\tau^{i}(x)\right)-\int_{0}^{1} \chi_{(c, d)}(y) d y\right|<\varepsilon
$$

if $p^{N-m^{\prime}}$ is sufficiently large, uniformly in $(c, d)$ and $x \in D$, where $\chi_{A}$ is the characteristic function of the set $A$. The same is true if $(c, d)$ is replaced by any closed or half-open interval. Thus (6) is true if $\chi_{(c, d)}$ is replaced by $\chi_{I}$ where $I$ is any interval, and also for step functions $h_{n}=\sum a_{i} \chi_{I_{i}}$, where the summation is over a finite number of terms. It follows that if $\sup _{n}\left(h_{n}(x)-h(x) \mid \rightarrow 0\right.$, where $h_{n}$ is a step function, then (6) is true with $\chi_{(c, d)}$ replaced by $h=\lim h_{n}$, i.e., if $x \in D$, and $h \in$ the space of regular functions which is the closure of the space of step-functions under the uniform norm, and includes the space of functions of BV. See [4, p. 139].

$$
\lim _{\substack{p^{N-m^{\prime} \rightarrow \infty} \\ x \in D}} \frac{1}{k} \sum_{k=1}^{k} h\left(\tau^{i}(x)\right)=\int_{0}^{1} h(x) d x \text {. Q.E.D. }
$$

3. Application to the irreducibility of certain nonnegative matrices. Consider the following class $\mathscr{A}$ of $n \times n$ matrices for various values of $n$. If $n$ is even then $A_{n} \in \mathscr{A}$ has the form

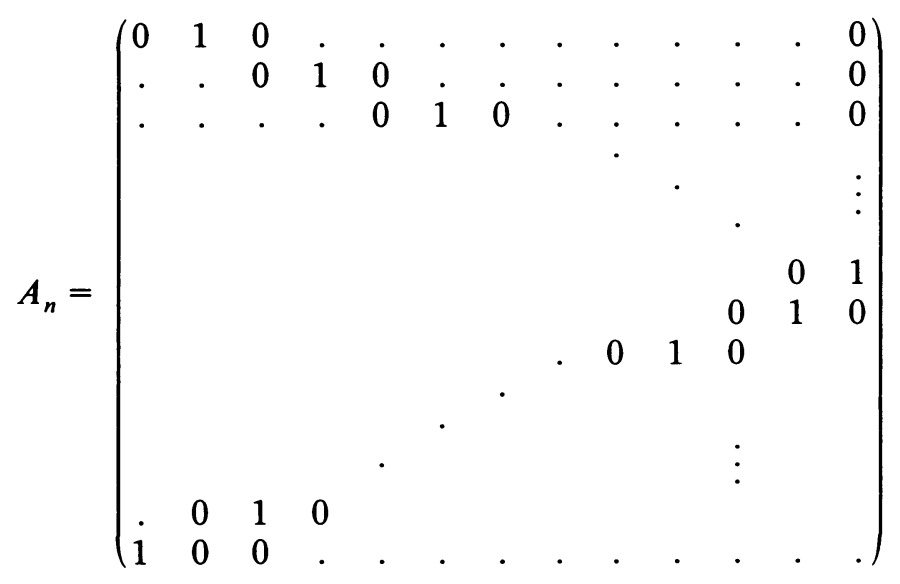


and if $n$ is odd, then $A_{n} \in \mathscr{A}$ has the form

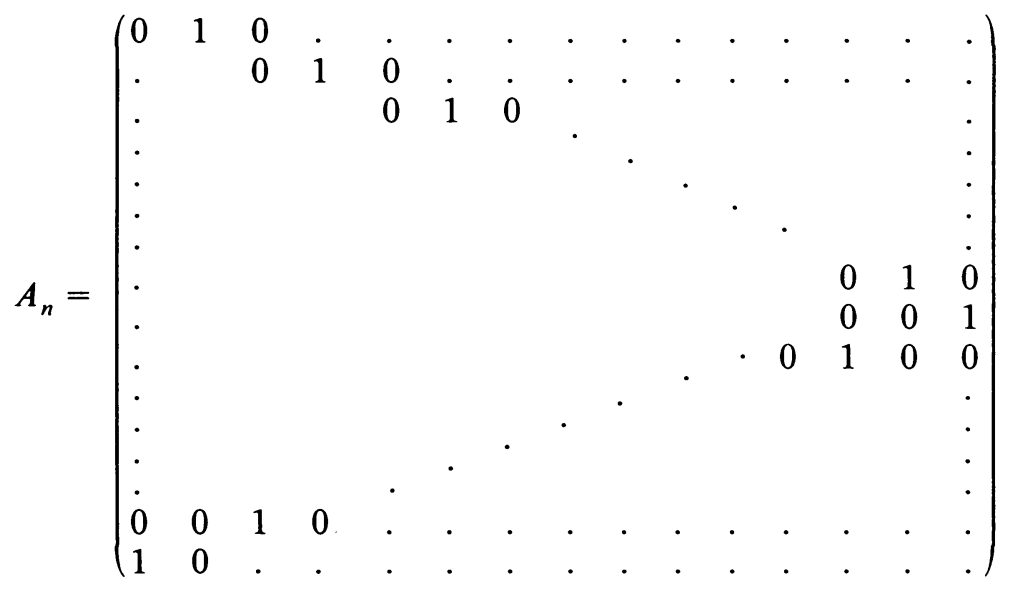

It is not hard to verify that $A_{2}$ and $A_{6}$ are irreducible, but that $A_{8}$ is reducible into two irreducible blocks. Also, $A_{3}, A_{5}, A_{9}, A_{11}$ are irreducible, but $A_{15}$ is reducible into 3 irreducible blocks. The problem is to determine the dependence of the irreducibility of $A_{n}$ on $n$. We shall present a partial solution to this problem using the results of $\$ 2$.

Let us associate with the map $f: D \rightarrow D$ a $0-1$ matrix $A$ where the $i j$ th entry is equal to 1 iff $f\left(2 i / p^{N}\right)=2 j / p^{N}, 1 \leqslant i, j \leqslant p^{N}-1$, and $(i, p)=(j, p)=1$ (i.e., we omit the $i$ th row or $j$ th column where $p$ divides $i$ or $j)$; otherwise the $i j$ th entry is 0 . $A$ is an $n \times n$ matrix where $n=((p-1) / 2) \cdot p^{N-1}$. If $(p-1) / 2$ is even, and $N=1$, then $A$ has the form (7), where $n=(p-1) / 2$.If $(p-1) / 2$ is odd, and $N=1$, then $A$ has the form (8), where $n=(p-1) / 2$.

Let $N=1$. Then $n=(p-1) / 2$ is the dimension of the square matrix $A_{n}$. Using the results in Table 1, we see from the fourth column how many periodic orbits correspond to a given $p$. Clearly, when there is only 1 such orbit, $A_{n}$ is irreducible. Hence for $n=1,2,3,5,6,9, \ldots, A_{n}$ is irreducible. But for $n=12,20,21,36, \ldots$, $A_{n}$ is reducible, with the number of irreducible blocks given in the fourth column of Table 1. Thus, $A_{n}$ can be permuted into a matrix with $d=(p-1) /(2 \cdot k)$ irreducible blocks (if $N>1$, then $\left.d=((p-1) /(2 \cdot k)) \cdot p^{N-1}\right)$. That is, there exists a permutation matrix $P$ such that

$$
P A P^{-1}=\left(\begin{array}{cccc}
A_{n}^{1} & & & \\
& A_{n}^{2} & & \\
& & \ddots & \\
& & & A_{n}^{d}
\end{array}\right) \text {, }
$$

where all the $A_{n}^{i}$ 's are irreducible matrices having the same dimension $k$ given in 
column 3 of Table 1. Each block $A_{n}^{i}$ corresponds to a period of $f$, say $a_{1} \rightarrow a_{2} \rightarrow$ $\cdots \rightarrow a_{k}$. We can label the rows and columns of $A_{n}^{i}$ so that each block looks like

$$
B_{n}^{i} \equiv\left(\begin{array}{cccccccccccc}
0 & 1 & 0 & . & . & . & . & . & . & . & . \\
0 & 0 & 1 & 0 & . & . & . & . & . & . & . \\
0 & 0 & 0 & 1 & 0 & . & . & . & . & . & . & . \\
& & & & & . & & & & & . \\
& & & & & & . & & & & . \\
0 & & & & & & & . & & & . \\
1 & 0 & . & . & . & . & . & . & . & 0 & 1 \\
& & . & & & 0 & 0
\end{array}\right) .
$$

Now this is a circulant matrix and its eigenvalues are the $k$ th roots of unity $[3, \mathrm{p}$. 309]. Thus $B_{n}^{i}$ has $x^{k}-1$ as its characteristic polynomial. Since $x^{k}-1$ has no repeated roots, $x^{k}-1$ is also the minimal polynomial. It follows that the characteristic polynomial of $A_{n}$ is $\left(x^{k}-1\right)^{d}$, and its minimal polynomial is $x^{k}-1$.

4. Concluding remarks. (i) All the foregoing results carry over to maps $g$ topologically conjugate to $\tau$, i.e., when there exists a homeomorphism $h:[0,1] \rightarrow[0,1]$ such that $g=h \circ \tau \circ h^{-1}$. An important example is the logistic map $g(x)=4 x(1-x)$ [2].

(ii) It is easy to show from the law of quadratic reciprocity that the number of periodic orbits is even iff $p=1+8 r$, where $r$ is any positive integer.

(iii) For $N=1$ the uniform distribution result of Theorem 2 does not necessarily hold as $p \rightarrow \infty$. Assuming, for instance, that there are an infinite number of primes of the form $p=2^{l} \pm 1$ (the primes of the form $2^{l}-1$ are known as the Mersenne primes), one sees easily that

$$
\alpha=\#\left\{m: \tau^{m}(2 / p) \in(c, d), 1 \leqslant m \leqslant k^{\prime}=l\right\}=\log (d / c)+O(1), \quad c>0 .
$$

Dividing through by $l$, we get $\alpha / l \rightarrow 0$ as $p \rightarrow \infty$. We conjecture that Theorem 2 and Corollary 1 still hold if we restrict $p$ to a class of primes such that $\lambda(p)$, the order of $2(\bmod p)$ is greater than $c \cdot p$, for some constant $c$.

(iv) Theorem 1 is true for $D_{n}=\{0<2 x / n<1, n$ odd, $(x, n)=1\}$, where $n=\prod_{i=1}^{m} p_{i}^{N_{i}}$. We conjecture that Theorem 2 is true if $\prod_{i=1}^{m} p_{i}^{N_{i}-m^{\prime},} \rightarrow \infty$. Also, since fractions of the form $x / 2^{a} n, a \geqslant 0,(x, 2)=1$ are clearly eventually periodic but not periodic, it follows that we have characterized the periodic behaviour of all rational points under the map $\tau$.

(v) If we take $f:[0,1] \rightarrow[0,1]$ to be the continuous map with $f(0)=0, f(1 / n)=1$, $f(2 / n)=0, \ldots$ so that $f$ has slopes $n,-n$, alternately, and $D=D_{N}=$ $\left\{a / p^{N},(a, p)=1\right.$, a even $\}$, where $p$ is an odd prime and $(p, n)=1$, then Theorem 1 still holds. It follows from well-known results about primtive roots; see e.g. [5, p. 50], that for any odd prime $p$ there exists an $n$ such that $n$ is a primitive root of $p^{N}$ for all $N$ and thus for this $n, D_{N}$ will consist exactly of one orbit $(N \geqslant 1)$. More interestingly, it follows from a recent result of Gupta and Murty [6] that there is an explicitly given set of 13 numbers - in fact, many such sets-such that one of those numbers, - call it $n$-is a primitive root of infinitely many primes. So for these primes, $f: D_{1} \rightarrow D_{1}$ consists of exactly one orbit. 


\section{REFERENCES}

1. R. May, Stability and complexity in model ecosystems, 2nd ed., Princeton Univ. Press, Princeton, N. J., 1978.

2. J. Guckenheimer, G. Oster and A. Ipaktchi, The dynamics of density dependent population models, J. Math. Bio. 4 (1977), 101-147.

3. B. Noble, Applied linear algebra, Prentice-Hall, Englewood Cliffs, N.J., 1969.

4. J. Dieudonné, Foundations of modern analysis, vol. 10, Academic Press, New York, 1960.

5. A. A. Gioia, The theory of numbers, Markham, Chicago, Ill., 1970.

6. R. Gupta and M. Murty, A remark on Artin's conjecture, Invent. Math. 78 (1984), 127-130.

Department of Mathematics, loyola Campus, Concordia University, Montreal, Quebec, CANADA H4B 1R6 